\title{
Tight software, methodological/physiological variation, less so
}

\author{
Henry Gewirtz, MDa \\ a Department of Medicine (Cardiology Division), Massachusetts General Hospital, Harvard \\ Medical School, Boston, MA
}

Received Dec 14, 2017; accepted Dec 14, 2017

doi: $10.1007 / \mathrm{s} 12350-018-1209-8$

\section{See related article, pp. 1912-1925}

\section{INTRODUCTION}

In this issue of the Journal $\mathrm{Wu}$ and co-workers report on the repeatability and reproducibility of PET ${ }^{11} \mathrm{C}$-acetate measurements of rest myocardial blood flow (MBFrst) and oxygen consumption (MVO2) in a small sample $(n=20)$ of clinically stable, primarily NYHA II patients with coronary artery disease (CAD) and heart failure reduced LVEF (HFrEF). PET measurements were combined with echo data to obtain derived indices of efficiency of $\mathrm{MVO} 2$ in production of external work; namely myocardial external efficiency (MEE) and work metabolic index (WMI). The patients were all studied at the authors' institution (Ottawa Heart Institute; OHI) as part of a larger study of patients with HFrEF and obstructive sleep apnea (OSA) though patients in the current study did not have OSA and their data were not included in the earlier report. ${ }^{1}$ Instead, the data were retrospectively analyzed to determine intra- and interobserver repeatability and reproducibility of PET ${ }^{11} \mathrm{C}$ acetate measurements of MBFrst and $\mathrm{MVO} 2$ and derived indices, MEE and WMI. Repeatability of all the measurements was assessed on average $\sim 1.5$ months post baseline, without overt change in patients' clinical condition. It is important to note, as the authors point out, half the patients were studied with one PET instrument, an older, PET-only device, with distinctly

Reprint requests: Henry Gewirtz, MD, Department of Medicine (Cardiology Division), Massachusetts General Hospital, Harvard Medical School, Yawkey 5E (Nuclear Cardiology), 55 Fruit St, Boston, MA 02114; hgewirtz@partners.org

J Nucl Cardiol 2018;25:1926-8.

1071-3581/ $\$ 34.00$

Copyright (c) 2018 American Society of Nuclear Cardiology. different and generally inferior performance characteristics compared with that of the other, a newer, PET-CT. PET data were analyzed independently using in house, largely automated software (FlowQuant) by 2 of the investigators. Briefly, the authors found that while intraand inter-observer correlations and repeatability for all parameters were excellent $\quad($ ICC $=0.95-0.99$ and $\mathrm{CR}=3-6 \%$ ), test-retest repeatability of patient data for various parameters ranged from $22 \%$ to $54 \%$ and as shown in Figure 5b was heavily instrument dependent with PET-CT device clearly outperforming the older PET-only scanner. Accordingly, the authors concluded (abstract):

11C-acetate PET measurements of WMI and MEE exhibited excellent test-retest repeatability and operator reproducibility. Newer PET-CT scanners may be preferred for longitudinal tracking of cardiac efficiency.

\section{COMMENTARY}

\section{Strengths}

The study has been performed by a group wellrecognized for long standing expertise and leadership in the field of cardiac PET. The data clearly demonstrate, in the authors' hands and using their highly automated software (FlowQuant ${ }^{\mathrm{TM}}$ ), that inter- and intra-observer reproducibility and repeatability of PET metrics of ${ }^{11} \mathrm{C}$ acetate, MBFrst and MVO2, and combined echo derived indices (myocardial external efficiency (MEE) and work metabolic index (WMI)) are excellent. Accordingly, the reader has good reason to be confident, as data obtained from the authors' laboratory in longitudinal clinical studies employing these metrics are unlikely to be confounded by data processing variation related either to intra- or inter-observer variability. It is likely, though unproven, that similar results could be obtained by other experienced investigators using similar though not 
necessarily the same highly automated software. The fact that method plus physiological reproducibility of MBFrst obtained with the PET-CT instrument ( 20\%) in the present study was comparable to that reported by others $^{2}$ for MBFrst measured with ${ }^{82} \mathrm{Rb}$ and different softwares (including details of tracer kinetic model) lends support to this notion.

The data obtained also provide limited information on the month-to-month repeatability (method plus physiological variation), of PET metrics of ${ }^{11} \mathrm{C}$-acetate indices of the efficiency of $\mathrm{MVO} 2$ in production of external work (MEE and WMI) in a very small sample of clinically stable, primarily NYHA class II patients with HFrEF due to ischemic cardiomyopathy (ICM). The authors are to be congratulated for presenting information illustrating the fact that the instrument employed matters considerably; a modern PET-CT is required for optimal repeatability in longitudinal studies (Figure 5b). The metric followed to assess MVO2 efficiency also appears to matter importantly. While the authors indicate that MEE and WMI have very similar non-parametric repeatability coefficient (each $\sim$ 19\%; Table 4) and so are equally useful, MEE appears distinctly more stable than WMI (respectively, $\sim 27 \%$ vs. $\sim 42 \%$; coefficient of repeatability $\pm 95 \%$ confidence interval; Figure 5a) and so indeed may be preferred as the authors themselves allude to.

\section{Limitations}

Sample size and composition are very important limitations which the authors recognize. Except for MEE, test-retest $\mathrm{CR} \pm 95 \%$ confidence interval for each of the major PET indices studied was substantially better for the PET-CT instrument in comparison with that of the PET-only scanner (Figure 5b). Thus, for purposes of longitudinal studies, the major conclusions of the study rest heavily on data from 9 patients studied with the PET-CT camera. Accordingly, the reader should be very cautious in extrapolating these results to the planning of similar longitudinal experiments involving $\mathrm{HFrEF}$ patients with ICM and prior coronary revascularization. It is likely that there will be lesser (i.e., worse) test-retest repeatability in a "sicker" HFrEF population predominately composed of NYHA Class III/IV ICM patients. Variation also may be different in another larger group of predominately Class II ICM. The sample size and composition of the present study is simply too small to make confident generalizations from (Figure 5a, b).

Though primarily a methods paper, the data illustrate an important physiological issue worthy of note. The ability to measure MBFrst and MVO2 with a single tracer injection makes the PET ${ }^{11} \mathrm{C}$-acetate a very attractive one. ${ }^{1,3-6}$ List mode data acquisition also makes possible measurement of $\mathrm{LV}$ end diastolic and systolic volumes as well as LV mass and hence MEE. ${ }^{7}$ Thus, parenthetically, any variation in test-retest repeatability related to non-simultaneous acquisition of PET and echo data alluded to by the authors potentially could have been mitigated had list mode acquisition of the PET data been obtained. Nevertheless, obtaining 2 indices of efficiency of $\mathrm{MVO} 2$ immediately raises questions of (A) whether one is superior to the other, (B) if they may potentially complement or conflict with one another when used longitudinally, and (C) how does each relate to MBFrst. The authors noted test-retest repeatability of MBFrst was not improved, and in fact worsened, when normalized to RPP; a not altogether unexpected finding given potential shortcomings of RPP as a surrogate for MVO2, ${ }^{8-10}$ since it fails to account for one of its major determinant, namely myocardial contractility. What is surprising is why they failed to normalize MBFrst to MVO2 which had been measured simultaneously in each patient. Table 3 indicates that the absolute values of MVO2 ( $\mathrm{mL} \cdot \mathrm{g} \cdot \mathrm{min})$ determined either by Kmono or $\mathrm{k} 2$ were very similar $(0.10 \pm 0.03$ vs. $0.09 \pm 0.02$, respectively) though the k2-based metric had arguably better CR $(\sim 37 \% \pm 95 \% \mathrm{CI})$ vs. that of Kmono ( 65\% $\pm 95 \%$ CI; Figure 5a) and so may have been the better choice based on this limited data set.

Another noteworthy physiological issue raised by the data analysis relates to the fact that both MBFrst and MVO2 were determined over the " ... whole ventricle (LV) mean (including normal and ischemic areas if present)....' Table 1 indicates $70 \%$ of patients had had PCI or CABG and that $65 \%$ prior myocardial infarction (MI). Accordingly, the data from physiologically very different regions of the heart were aggregated together and analyzed as if they were the same, which clearly, they were not. Thus, the absolute values both of global MBFrst and MVO2 may be uninformative and potentially misleading in these patients. It would have been far better to have carried the analysis forward with physiologically similar segments (e.g., infarct, impaired, and normal flow reserve) grouped together. Not only would such analysis provide a more meaningful estimate of absolute values of MBFrst and MVO2 and the relationship between the two but also whether test-retest repeatability was comparable, or not, for each.

\section{SUMMARY/CONCLUSIONS}

The data provided in the current paper clearly indicate that it is possible with highly automated software to obtain excellent intra- and inter-observer test-retest repeatability and reproducibility of PET ${ }^{11} \mathrm{C}$ acetate measurements of global LV MBFrst, MVO2 and derived indices MEE and WMI in clinically stable, 
NYHA class II, HFrEF patients. Further, rather substantial variation in test-retest repeatability of these parameters, on the order of $25 \%-40 \%(\mathrm{CR} \pm 95 \% \mathrm{CI}$; Figure 5a), appears to be related to some combination of purely methodological and physiological factors, notwithstanding, respectively, superior technical expertise of the laboratory and apparent clinical stability of the patients. A non-parametric estimate of MEE and WMI, test-retest repeatability was on the order of $20 \%$ (NPC, Table 4) and perhaps is more applicable to very small data sets, which may not conform to a normal distribution. Finally, a note of caution concerning the absolute values of global averages of MBFrst, MVO2 and derived indices MEE and WMI all of which may be very misleading in the left ventricles of patients with revascularized ICM and prior MI. Global metrics by definition will average away differences in myocardial regions which may have very different physiological characteristics and so should be considered separately.

\section{Disclosure}

None.

\section{References}

1. Hall AB, Ziadi MC, Leech JA, Chen SY, Burwash IG, Renaud J, et al. Effects of short-term continuous positive airway pressure on myocardial sympathetic nerve function and energetics in patients with heart failure and obstructive sleep apnea: A randomized study. Circulation. 2014;130(11):892-901.

2. Kitkungvan D, Johnson NP, Roby AE, Patel MB, Kirkeeide R, Gould KL. Routine clinical quantitative rest stress myocardial perfusion for managing coronary artery disease: Clinical relevance of test-retest variability. JACC Cardiovasc Imaging. 2017;10(5):565-77.

3. Beanlands RS, Nahmias C, Gordon E, Coates G, deKemp R, Firnau G, et al. The effects of beta(1)-blockade on oxidative metabolism and the metabolic cost of ventricular work in patients with left ventricular dysfunction: A double-blind, placebo-controlled, positron-emission tomography study. Circulation. 2000;102(17):2070-5.

4. Porenta G, Cherry S, Czernin J, Brunken R, Kuhle W, Hashimoto $\mathrm{T}$, et al. Noninvasive determination of myocardial blood flow, oxygen consumption and efficiency in normal humans by carbon11 acetate positron emission tomography imaging. Eur $\mathrm{J}$ Nucl Med. 1999;26(11):1465-74.

5. Ukkonen H, Beanlands RS, Burwash IG, de Kemp RA, Nahmias C, Fallen E, et al. Effect of cardiac resynchronization on myocardial efficiency and regional oxidative metabolism. Circulation. 2003;107(1):28-31.

6. Yoshinaga K, Burwash IG, Leech JA, Haddad H, Johnson CB, deKemp RA, et al. The effects of continuous positive airway pressure on myocardial energetics in patients with heart failure and obstructive sleep apnea. J Am Coll Cardiol. 2007;49(4):450-8.

7. Hansson NH, Tolbod L, Harms J, Wiggers H, Kim WY, Hansen E, et al. Evaluation of ECG-gated [(11)C]acetate PET for measuring left ventricular volumes, mass, and myocardial external efficiency. J Nucl Cardiol. 2016;23(4):670-9.

8. Kal JE, Van Wezel HB, Vergroesen I. A critical appraisal of the rate pressure product as index of myocardial oxygen consumption for the study of metabolic coronary flow regulation. Int J Cardiol. 1999;71(2):141-8.

9. Moffitt EA, Sethna DH, Gray RJ, DeRobertis M, Matloff JM, Bussell JA. Rate-pressure product correlates poorly with myocardial oxygen consumption during anaesthesia in coronary patients. Can Anaesth Soc J. 1984;31(1):5-12.

10. Nordlander R, Pehrsson SK, Astrom H. The reliability of ratepressure product as an index of myocardial oxygen consumption in atrial synchronized versus fixed rate ventricular pacing. Pacing Clin Electrophysiol. 1989;12(6):917-21. 